\title{
Electroconvection in a dielectric liquid between two concentric half-cylinders with rigid walls: Linear and nonlinear analysis
}

\author{
Pedro A. Vázquez* \\ Departamento de Física Aplicada III, ETSII, Universidad de Sevilla, Camino de los Descubrimientos s/n, 41092 Sevilla, Spain \\ Alberto T. Pérez \\ Departamento de Electrónica y Electromagnetismo, Facultad de Física, Universidad de Sevilla, Avda. de Reina Mercedes s/n, \\ 41013 Sevilla, Spain \\ Philippe Traoré \\ Institut PPRIME, Département Fluide-Thermique-Combustion, Boulevard Pierre et Marie Curie, BP 30179, \\ 86962 Futuroscope-Chasseneuil, France \\ Jian $\mathrm{Wu}^{\dagger}$ \\ School of Energy Science and Engineering, Harbin Institute of Technology, Harbin 150001, People's Republic of China
}

(Received 2 October 2017; published 20 February 2018)

\begin{abstract}
We study the linear stability and nonlinear behavior of the electroconvection between two concentric halfcylinders with no-slip conditions on all boundaries. The no-slip condition makes impossible to apply the standard modal approach. Hence, we apply a finite element technique similar to the one we have used in a previous paper about the electroconvection in a rectangular enclosed domain. When compared to the classical problem of electroconvection between two full concentric cylinders, the linear criterion is higher, due to the viscous shear introduced by the lateral walls. As a consequence, the structure of the eigenmodes is very different. There is a repulsion between modes with the same symmetry, forcing pairs of modes to cross each other repeatedly. For inner injection and small value of the ratio between the inner and outer radii the no-slip condition changes the nature of the bifurcation from subcritical to supercritical, while it is always subcritical for outer injection. To understand this behavior, we perform a modal analysis using the eigenfunctions obtained from the linear stability analysis as modal basis. We show that the supercritical branch is originated by the nonorthogonality of the modes when no-slip boundary conditions are imposed. These mechanism explains the previously unexplained appearance of the supercritical branch in the enclosed rectangular configuration.
\end{abstract}

DOI: 10.1103/PhysRevE.97.023106

\section{INTRODUCTION}

We study the linear stability and the nonlinear bifurcation of the electrohydrodynamic (EHD) convection in a dielectric liquid between two half-cylinders with no-slip boundary conditions in the strong injection regime. EHD is an interdisciplinary science dealing with the interaction of fluids and electric fields. It has a great number of applications in industrial processes: EHD pumping [1-3], heat transfer enhancement [4-6], turbulent mixing [7], EHD spraying and atomization [8,9], and biomicroelectromechanical systems (bio-MEMS) and nanotechnology applications [10-12].

One of the classical problems in EHD is the convection generated between two infinite cylindrical and concentric electrodes with radii $R_{i}<R_{o}$. The space between the cylinders is filled with a perfectly dielectric liquid. An electric potential difference is applied between the cylinders. When the applied voltage is above a critical value, injection on one or both of the

\footnotetext{
*pvazquez@us.es

†jian.wu@hit.edu.cn
}

electrodes occurs. We will consider only unipolar injection, inner if the injecting electrode is the one with radius $R_{i}$ and outer if it is the one with radius $R_{o}$. The injected charge having the same polarity as the injecting electrode, the ions are pushed away from the electrode, setting the liquid into motion. The linear stability analysis of the full 3D problem was performed by Agräit and Castellanos [13]. In a recent paper, Fernandes et al. [14] studied the linear stability of this problem, both numerically and analytically, although they did not considered the nonlinear regime.

In a previous paper [15], the authors studied numerically the linear stability and the nonlinear bifurcation of the full cylinder problem simplified to a $2 \mathrm{D}$ configuration. The bifurcation is subcritical, that is, once the critical voltage is attained, the stationary branch is unstable. The velocity of the fluid increases until, in the final steady state, the maximum velocity of the liquid is greater than the drift velocity of the ions. The charge distribution is then controlled by the velocity field and regions void of charge appear. This nonlinear bifurcation is similar to what happens in one of the most classical problems in EHD, the convection generated by two infinite parallel electrodes immersed in a perfectly dielectric liquid and subjected to a 
electric voltage applied between the electrodes. However, in two recent papers $[16,17]$ the authors considered a variation of this two-plate problem, with the infinite domain substituted by a finite closed rectangular domain with no-slip boundary conditions applied on all the boundaries. In the first paper, using numerical simulations, the authors showed that the bifurcation may become supercritical for some values of the aspect ratio of the domain. In this case, steady convective rolls with maximum velocity smaller than the drift velocity of the ions can be formed. In the second paper the authors studied the linear stability of the enclosed problem, and showed that the structure of the modes is quite different from that of the infinite case.

Here, we study a similar modified version of the twocylinder configuration. We consider the electroconvection between two concentric half-cylinders, of radii $R_{i}<R_{o}$ with no-slip boundary conditions on all the boundaries. An electric voltage is applied between the half-cylinders, with the inner cylinder connected to a higher voltage and the outer one grounded for inner injection, and the reversed way for outer injection. We will assume that unipolar injection takes place in both cases. We study the linear stability of this problem, along with the nature of the nonlinear bifurcation. We restrict our analysis to the strong injection regime, although the main conclusions are applicable to arbitrary injection strength. The change of geometry from the planar one introduces two new elements influencing the system: the injection direction and the radius ratio. We will see that the behavior of the system is different when the injection of charge occurs at the inner or the outer electrodes. This is especially true for the change of the nature of the bifurcation from subcritical to supercritical. This different behavior offers some clues of the explanation of this change of nature.

The results of the linear stability analysis are similar to those obtained in Pérez et al. [17]. The no-slip boundary conditions increases the threshold value for injection and changes the structure of the modes. The modes with the same symmetry repel each other as the ratio between the inner and outer radii changes. For the nonlinear aspects, the presence of no-slip walls can change the nature of the bifurcation, as it was discussed by the authors in $\mathrm{Wu}$ et al. [16] in a rectangular domain with top and bottom flat electrodes. With free-slip lateral walls the bifurcation is always subcritical. When no-slip boundary conditions are imposed the bifurcation becomes supercritical for some ranges of values of $\Gamma$. In the half-cylinder configurations, the ratio between the radii of the inner and outer cylinders plays the role of the aspect ratio in the rectangular configuration. When free-slip conditions are imposed on the flat walls the bifurcation is always subcritical, as it is the case with full concentric cylinders [15]. When no-slip conditions are imposed, and the injection of ions occurs on the inner electrode, the bifurcation may become supercritical for small values of the radii ratio. As this ratio is increased it becomes subcritical again and remains that way. However, when the injection occurs at the outer electrode, the bifurcation is subcritical for all the values of the radii ratio that we have explored.

We have performed a modal analysis of the evolution of the flow pattern using the eigenmodes for each configuration computed in the linear stability analysis as a basis. In the free-slip case the modes are essentially orthogonal, while in the no-slip configuration they are not. We show that, for inner injection, the supercritical nature of the bifurcation can be explained by this nonorthogonality, as several modes can extract energy from the electric field and their final amplitudes are of similar values. The bifurcation becomes subcritical when the second mode is excited. This mode modifies the charge distribution and throws the system away from the supercritical branch into the upper one. In the outer injection case, the modes are still nonorthogonal, but the critical value of the stability parameter is much higher than in the inner injection regime. As a consequence, the modes grow so much that the velocity field takes control of the distribution of electric charge and a region void of charge is created, which is a characteristic of the subcritical bifurcation. This mechanism explains also the previously unexplained appearance of the supercritical branch in the closed rectangular configuration described in reference Pérez et al. [17].

The paper is organized as follows. In Sec. II we state the physical domain, the governing equations, the boundary conditions of both free- and rigid-walls cases and inner and outer injection configurations. The relevant nondimensional parameters are also set. Section III is devoted to the description of the method dealing with the linear stability analysis, as well as analyzing the results. As it was the case in Pérez et al. [17], the no-slip boundary conditions on the flat walls prevents the use of the standard modal analysis used by Agräit and Castellanos [13]. A semianalytical technique based on finite elements is applied. In Sec. IV we examine the nonlinear behavior. First, as a test of the numerical technique, we present the linear stability criteria obtained from the simulations and compare them to the values obtained from the eigenvalue analysis. After that, we discuss the nature of the bifurcation for each configuration. Finally, we perform a modal analysis to understand the nonlinear behavior for each configuration. The main conclusions are summarized in Sec. V.

\section{PROBLEM FORMULATION}

\section{A. Basic equations and boundary conditions}

We consider a dielectric liquid confined in the $2 \mathrm{D}$ region between two-half cylinders, of radius $R_{i}$ and $R_{o}$, with $R_{i}<R_{o}$ (see Fig. 1). The liquid is assumed to be incompressible, Newtonian, isothermal, and perfectly insulating, with mass density $\rho$, kinematic viscosity $v$, and absolute permittivity $\varepsilon$. When inner injection is considered, a stationary electric potential $\Phi_{a}$ is applied to the inner cylinder and the outer is grounded. The applied potentials are reversed when the outer injection case is studied. The electric field acts upon these charges and, if the applied potential is high enough, put the liquid in motion. We will make the assumption of unipolar injection, where only one of the electrodes injects charges and only one type of charge carriers is present. We will further assume that the density of injected charge on the injecting electrode, $q_{i}$, is constant (autonomous injection). The collecting electrode is assumed to behave as an open boundary for the ions. These are common assumptions in EHD problems [18].

The constitutive law for the current density is

$$
\boldsymbol{J}=q K \boldsymbol{E}-D \nabla q+q \boldsymbol{u},
$$

where $q$ is the volumetric charge density, $\boldsymbol{u}$ is the fluid velocity, and $\boldsymbol{E}$ is the electric field. The first term in Eq. (1) is the 




FIG. 1. Computational domain with dimensional boundary conditions for inner injection. For outer injection the boundary conditions on the electric potential are interchanged and the boundary condition for the charge density is applied at the outer electrode.

drift current, $K$ being the ionic mobility of the charge carriers. The second term is the contribution from diffusion where $D$ is the charge diffusion coefficient. The last term is due to the convection of the electric charge by the velocity of the liquid $\boldsymbol{u}$. In EHD problems, usually diffusion is negligible compared to drift [18]. Hence, the charge conservation equation becomes:

$$
\frac{\partial q}{\partial t}+\nabla \cdot[q(K \boldsymbol{E}+\boldsymbol{u})]=0 .
$$

As boundary condition for the charge we impose $q=q_{i}$ on the

$$
\begin{array}{ll}
r=\bar{R}_{i}=\frac{\Gamma}{1-\Gamma}: & \Phi=1 \text { (inner), } \\
& \Phi=0 \text { (outer), } \\
r=\bar{R}_{o}=\frac{1}{1-\Gamma}: & \Phi=0 \text { (inner), } \\
\theta=0, \pi: & \Phi=1 \text { (outer), } \\
\theta & \partial / \partial y=0
\end{array}
$$

Here, $\bar{R}_{i}=R_{i} / d, \bar{R}_{o}=R_{o} / d$, and $\Gamma=R_{i} / R_{o}$. Figure 2 shows the geometry and boundary conditions of the problem in nondimensional form. In the rest of the paper we will use the terms lateral walls or flat sides to describe the $\theta=0, \pi$ boundaries.

The nondimensional parameters appearing in the equations and boundary conditions are

$$
T=\frac{\varepsilon \Phi_{a}}{\rho \nu K}, \quad C=\frac{q_{i} d^{2}}{\varepsilon \Phi_{a}}, \quad M=\frac{1}{K}\left(\frac{\varepsilon}{\rho}\right)^{1 / 2}, \quad \Gamma=\frac{R_{i}}{R_{o}}
$$

The parameter $T$ is the ratio between the electric and viscous terms in Navier-Stokes equation. It acts as a kind of electric Rayleigh number and will stand for the stability parameter. The mobility number $M$ is the ratio between the so called hydrodynamic mobility and the ionic mobility [19]. It only depends on the fluid properties. The injection number $C$ injecting electrode. Figure 1 shows the dimensional boundary conditions for inner injection.

The equations describing the problem are the Poisson equation, the Navier-Stokes equations, and the charge conservation equation. To build the nondimensional equations we choose the following scales:

$$
\begin{array}{lll}
x, y \sim d=\left(R_{o}-R_{i}\right), & t \sim d^{2} / K \Phi_{a}, & u \sim K \Phi_{a} / d, \\
\Phi \sim \Phi_{a}, & E \sim \Phi_{a} / d, & q \sim \varepsilon \Phi_{a} / d^{2}, \\
p \sim \varepsilon \Phi_{a}^{2} / d^{2} . & &
\end{array}
$$

We have used the distance between the cylinders, $d=R_{o}-R_{i}$, to define the spatial scale. With these choices, the nondimensional equations of the problem become

$$
\begin{aligned}
& \frac{\partial \boldsymbol{u}}{\partial t}+(\boldsymbol{u} \cdot \nabla) \boldsymbol{u}=-M^{2} \nabla p+\frac{M^{2}}{T} \Delta \boldsymbol{u}+M^{2} q \boldsymbol{E}, \\
& \nabla \cdot \boldsymbol{u}=0 \\
& \Delta \Phi=-q, \\
& \boldsymbol{E}=-\nabla \Phi, \\
& \frac{\partial q}{\partial t}+\nabla \cdot[q(\boldsymbol{u}+\boldsymbol{E})]=0 .
\end{aligned}
$$

As the fluid is considered to be isothermal, the dielectric force is not relevant. The electrostriction force, being the gradient of a scalar magnitude, can be included in the gradient of the pressure. Therefore, the force term in the first equation in Eq. (4) is restricted to the Coulomb force [18].

The nondimensional boundary conditions are

$$
\begin{array}{lc}
u_{x}, u_{y}=0, & q=C \text { (inner), } \\
u_{x}, u_{y}=0, & \\
& q=C \text { (outer), } \\
u_{x}, u_{y}=0 & \text { (rigid walls) } \\
\partial u_{x} / \partial y, u_{y}=0 & \text { (free walls). }
\end{array}
$$

measures the injection strength. In this paper we will only consider the strong injection case $(C=10)$.

\section{LINEAR STABILITY ANALYSIS}

We study here the linear stability of the hydrostatic state. We apply the same technique detailed in Pérez et al. [17], adapted to the half-cylinders geometry. For small values of the applied voltage viscosity prevents any motion of the liquid. As the voltage is increased, the liquid is eventually put in motion when a certain threshold value is attained. We study the linear stability of the system using the standard method of small perturbations. The steady state is perturbed with small perturbations and we study how they grow. If all perturbations decay, the system is stable. The smallest value of the stability parameter for which the perturbations grow in time is the critical value $T_{c}$. 


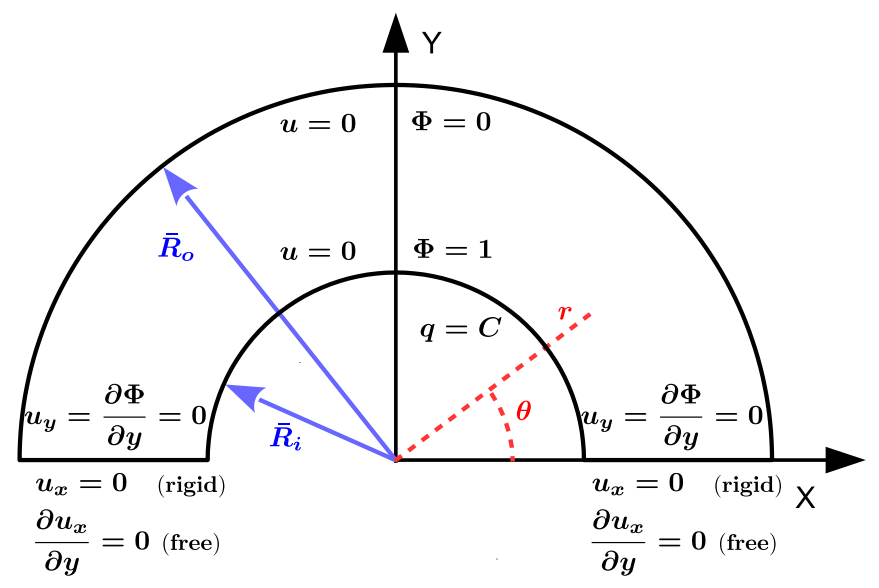

FIG. 2. Computational domain with nondimensional boundary conditions for inner injection. For outer injection the boundary conditions on the electric potential are interchanged and the boundary condition for the charge density is applied at the outer electrode.

\section{A. Hydrostatic solution}

The hydrostatic solution corresponds to a steady state characterized by the liquid at rest. The hydrostatic profiles of the charge and the electric field can be computed from equations Eq. (4) imposing that the temporal derivatives are zero and the velocity $\boldsymbol{U}_{0}=\mathbf{0}$. The equations for the hydrostatic state are

$$
\begin{aligned}
-\nabla P_{0}+Q_{0} \boldsymbol{E}_{0} & =0, \\
\Delta \Phi_{0} & =-Q_{0}, \\
\boldsymbol{E}_{0} & =-\nabla \Phi_{0}, \\
\nabla \cdot\left(Q_{0} \boldsymbol{E}_{0}\right) & =0 .
\end{aligned}
$$

The solutions only depend on the radial coordinate, hence the electric field can be expressed as $\boldsymbol{E}_{0}=E_{0}(r) \boldsymbol{e}_{r}$ and the charge profile as $Q_{0}=Q_{0}(r)$. The equations are

$$
\begin{aligned}
\frac{1}{r} \frac{d\left(r E_{0}\right)}{d r} & =Q_{0}, \\
\frac{d\left(r Q_{0} E_{0}\right)}{d r} & =0 .
\end{aligned}
$$

The boundary conditions are

$$
\begin{aligned}
& \text { inner injection: } Q_{0}\left(r=\bar{R}_{i}\right)=C, \quad \int_{\bar{R}_{i}}^{\bar{R}_{o}} E_{0} d y=1, \\
& \text { outer injection: } \quad Q_{0}\left(r=\bar{R}_{o}\right)=C, \quad \int_{\bar{R}_{o}}^{\bar{R}_{i}} E_{0} d y=1 .
\end{aligned}
$$

The solutions have the form

$$
\begin{aligned}
& E_{0}(r)=\frac{R_{c} E_{s}}{r} \sqrt{1+\frac{C}{E_{s} R_{c}}\left(r^{2}-R_{c}^{2}\right)}, \\
& Q_{0}(r)=\frac{C}{\sqrt{1+\frac{C}{E_{S} R_{c}}\left(r^{2}-R_{c}^{2}\right)}} .
\end{aligned}
$$

Here, $R_{c}=\bar{R}_{i}\left(\bar{R}_{o}\right)$ stands for inner (outer) injection. $E_{s}$ is the value of $E_{0}$ at the injector. It is defined by Eq. (9), which is an implicit equation in $E_{s}$. This equation can be solved by numerical integration for each value of $C . E_{s}$ is positive for inner injection and negative for outer injection.

\section{B. Perturbation equations}

We look for perturbations of the base magnitudes. We express the physical magnitudes as

$$
\begin{aligned}
& \boldsymbol{u}(x, y, t)=\boldsymbol{u}^{\prime}(x, y, t), \\
& p(x, y, t)=P_{0}(x, y)+p^{\prime}(x, y, t), \\
& \Phi(x, y, t)=\Phi_{0}(x, y)+\Phi^{\prime}(x, y, t), \\
& \boldsymbol{E}(x, y, t)=\boldsymbol{E}_{0}(x, y)+\boldsymbol{E}^{\prime}(x, y, t), \\
& q(x, y, t)=Q_{0}(x, y)+q^{\prime}(x, y, t) .
\end{aligned}
$$

The primed quantities are supposed to be much smaller than the corresponding base quantities. Introducing these expressions into Eq. (4), taking into account Eqs. (7), and keeping only the first order terms in the primed quantities leads to the following set of equations:

$$
\begin{aligned}
& \frac{\partial \boldsymbol{u}^{\prime}}{\partial t}=-M^{2} \nabla p^{\prime}+\frac{M^{2}}{T} \Delta \boldsymbol{u}^{\prime}+M^{2}\left(Q_{0} \boldsymbol{E}^{\prime}+q^{\prime} \boldsymbol{E}_{0}\right), \\
& \nabla \cdot \boldsymbol{u}^{\prime}=0, \\
& \Delta \Phi^{\prime}=-q^{\prime}, \\
& \boldsymbol{E}^{\prime}=-\nabla \Phi^{\prime}, \\
& \frac{\partial q^{\prime}}{\partial t}+\nabla \cdot\left[Q_{0}\left(\boldsymbol{u}^{\prime}+\boldsymbol{E}^{\prime}\right)+q^{\prime} \boldsymbol{E}_{0}\right]=0 .
\end{aligned}
$$

The standard way to proceed is to write the primed equations as a product of an exponential function depending on time and a function depending on the spatial variables alone,

$$
\left(\begin{array}{c}
\boldsymbol{u}^{\prime} \\
p^{\prime} \\
\Phi^{\prime} \\
q^{\prime}
\end{array}\right)=\left(\begin{array}{l}
\boldsymbol{U}(x, y) \\
\Pi(x, y) \\
V(x, y) \\
Q(x, y)
\end{array}\right) e^{\sigma t}
$$

The symbol $\sigma$ denotes the growth rate, which can be complex. When $\operatorname{Im}(\sigma)=0$, the principle of exchange of stabilities applies. As it is discussed in Atten and Moreau [20] for the plane-plane geometry, it is not possible to prove rigorously that this principle applies in the free walls case. But the numerical results show that this is actually the case. Here, we will assume that $\operatorname{Im}(\sigma)=0$ for both free and rigid walls situations. Then, for the marginal state that corresponds to the onset of the motion it is $\sigma=0$. The comparison with the results issued from the direct numerical computation will validate this assumption.

In the free walls case, when symmetric boundary conditions are applied at the flat sides, the spatial functions can be developed in terms of normal modes, adapting the 3D analysis in Agräit and Castellanos [13] to the 2D geometry. As an example, the charge perturbation would be expressed, in polar coordinates, as $Q(r, \theta)=f(r) e^{i m \theta}$, the number $m$ being related to the number of rolls. The no-slip boundary conditions on the flat sides prevent us from applying the same procedure in the rigid walls case, and a different strategy based on a numerical computation must be envisaged. 
We introduce Eqs. (13) into Eqs. (12). We are interested in the margin stability, $\sigma=0$, so the time derivative does not appear. In this way, the number $M$ disappears too and will have no influence on the linear stability. The pressure can be rescaled to include the parameter $T$ in its definition. We also drop for convenience the primes from the magnitudes. Then the stability equations can be rewritten as

$$
\begin{aligned}
& \nabla p-\Delta \boldsymbol{u}=T\left(-Q_{0} \nabla V+q \boldsymbol{E}_{0}\right), \\
& \nabla \cdot \boldsymbol{u}=0, \\
& \Delta V=-q, \\
& \nabla \cdot\left[Q_{0}(\boldsymbol{u}-\nabla V)+q \boldsymbol{E}_{0}\right]=0 .
\end{aligned}
$$

The boundary conditions are now

$$
\begin{aligned}
& r=\bar{R}_{i}=\frac{\Gamma}{1-\Gamma}: V=0, \quad u_{x}, u_{y}=0, \quad \text { inner injection }: q=0, \\
& r=\bar{R}_{o}=\frac{1}{1-\Gamma}: V=0, \quad u_{x}, u_{y}=0, \quad \text { outer injection : } q=0, \\
& \theta=0, \pi: \quad \partial V / \partial y=0, \quad u_{x}, u_{y}=0 \quad \text { (rigid walls), } \\
& \partial u_{x} / \partial y, u_{y}=0 \quad \text { (free walls). }
\end{aligned}
$$

Equations (14) along with boundary conditions Eqs. (15) define an eigenvalue problem. The secular determinant leads to a relation between the relevant parameters of the form

$$
\mathcal{F}(T, C, \Gamma)=0 .
$$

We take $T$ as the stability parameter, that is, for $C$ and $\Gamma$ fixed, the different values of the eigenvalue $T$ give the excitation thresholds of the different modes. The smallest of these eigenvalues, $T_{c}$, will be the threshold for the linear stability.

The generalized eigenvalue problem is solved using finite elements. To build the matrices, we use DOLFIN [21], an interface to the FEniCS package [22]. This is a framework for automated solution of partial differential equations based on the Finite Element method. The package allows the construction of the relevant matrices using a high level formulation of the weak problem. It also handles the computation of eigenvalues and eigenfunctions through the SLEPc package [23], a software library for the solution of large scale sparse eigenvalue problems. We refer to Pérez et al. [17] for more details.

\section{Results}

We have considered the case $C=10$, both with free and rigid walls, for both inner and outer injection. We have used triangular meshes built over a $200 \times 100$ structured grid, that is, with 40000 triangular elements. The meshes are thinner near the injecting half-cylinder, and coarser next to the collecting one.

\section{Free walls case}

We use the free walls case as a validation of our method. In this configuration the standard modal expansion can be used to study the linear stability. Let us point out that, when free-slip boundary conditions are imposed on the flat sides in Fig. 2, the linear stability problem becomes equivalent to the complete cylinders configuration. Agräit and Castellanos [13] studied the linear stability of the 3D configuration with whole cylinders by applying the classical modal expansion.
We have adapted their study to the $2 \mathrm{D}$ case. We have solved the resulting eigenvalue problem using the bvp $4 \mathrm{c}$ function in MATLAB. This function implements a collocation method with $\mathrm{C} 1$ piecewise cubic polynomials [24].

Figure 3 shows the critical value $T_{c}$ as a function of $\Gamma$ for inner and outer injection obtained with the standard modal analysis and the one developed here for purpose based on finite elements. The results obtained with the two different methods are in excellent agreement. In the strong injection regime under consideration, inner injection is always less stable than outer injection for all $\Gamma$. In addition, as $\Gamma$ approaches 1 , the critical value for both injection configurations tends towards the critical value for infinitely large plate-plate case, $T_{c}^{p}=164.1$ [20]. The results of the FE method for $\Gamma$ very close to 1 are not provided. The reason is that, as $\Gamma \rightarrow 1$, the computational

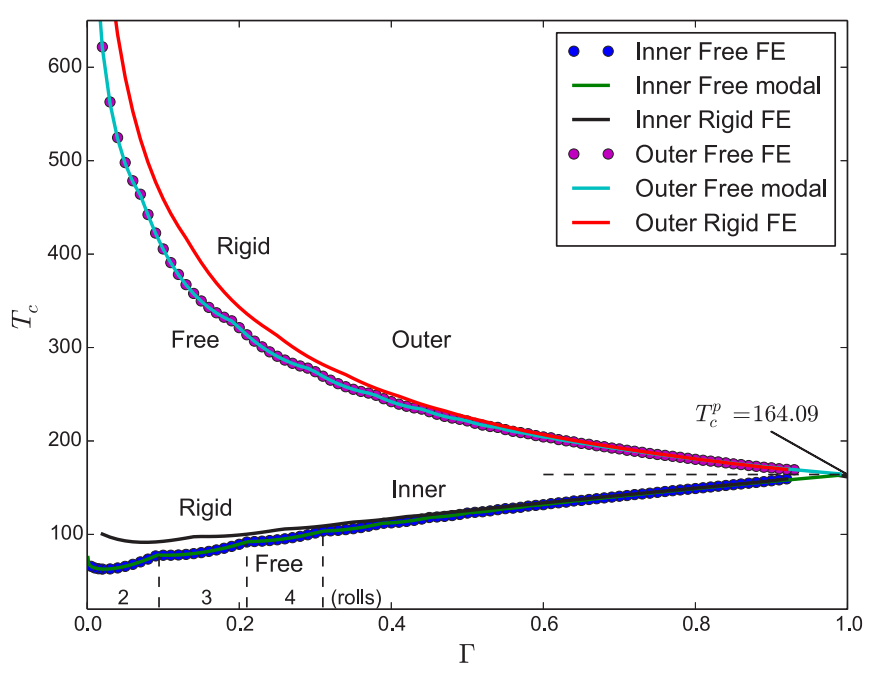

FIG. 3. Linear threshold computed with Finite Elements (FE) and modal analysis for inner and outer injection for $C=10$. The results computed with FE for rigid walls are also plotted. As $\Gamma$ approaches 1 , the value of $T_{c}$ goes to the critical value corresponding to the plane case, $T_{c}^{p}$, for all the cases. The number of rolls corresponding at each hump is indicated for the inner injection branch in the free walls case. 


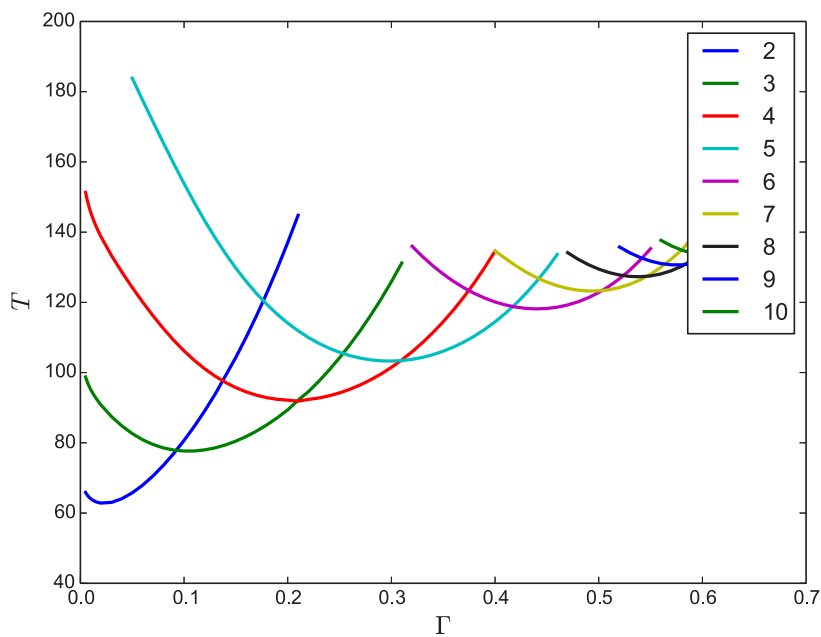

(a)

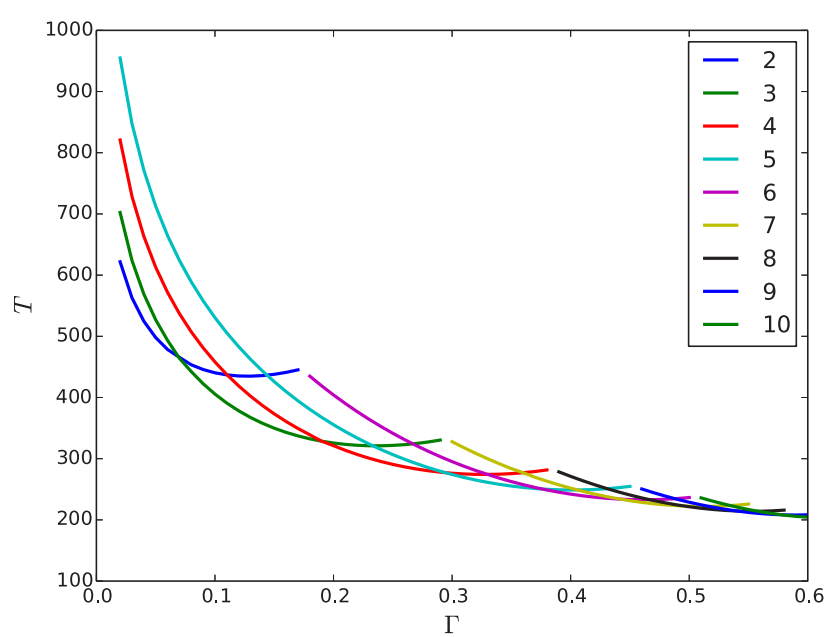

(b)

FIG. 4. Eigenvalues of the first four modes vs. $\Gamma$ for the free walls case for inner injection (a) and outer injection (b). The numbering in the legend corresponds to the number of rolls observed in the half-cylinders configuration. Only a portion of each mode is showed.

domain becomes very large, considering $\bar{R}_{i}=\Gamma /(1-\Gamma)$ and $\bar{R}_{o}=1 /(1-\Gamma)$. Thus, a larger number of elements is required and the computation time increases dramatically. Anyway, for values of $\Gamma$ close to 1 the results are similar to those obtained for a planar geometry.

In Fig. 3 we can observe some humps in both branches. Each hump corresponds to a different number of velocity rolls for the velocity field (related to the $m$ value in the modal analysis [13]). The number of rolls observed in the half-cylinder geometry are indicated up to 4 for the inner injection case. Figure 4 shows the eigenvalues of the first four modes for each value of $\Gamma$. The cusps in the lower branch of Fig. 3 corresponds to the crossing of two different modes as displayed in Fig. 4. Note that we have plotted only a portion of each mode. If we prolong the branches all the modes cross each other, although at values of $T$ higher than the critical value for the corresponding value of $\Gamma$.

We have plotted in Fig. 5 the eigenfunctions of the most unstable modes in the inner injection case for $\Gamma=0.05$ and $\Gamma=0.15$. The former corresponds to a point inside the first hump in Fig. 3, while the latter lies inside the second hump. The stream function for the first mode is antisymmetric with respect to the $x=0$ line, while the second mode is symmetric. This pattern repeats itself with higher modes. Also, all the velocity rolls are equivalent, in the sense that they correspond to the modal factor $e^{i m \theta}$ in the modal expansion. They only adapt their sizes to the total number of rolls present and the space available between the electrodes.

The crossing of the modes for the free walls case is similar to the behavior in the planar injection problem discussed in Pérez et al. [17]. However, there is a fundamental difference in the interpretation of the results. In the planar case, the free walls configuration is a way of modeling two infinite parallel planes. In this problem, all the modes have the same minimum value of the linear threshold $T_{c}^{p}=164.1$. In a real experiment with a sufficiently large domain, the system would choose this absolute minimum value as the linear stability threshold. In the cylinder configuration the situation is different. Indeed, if we could perform an experiment keeping the $2 \mathrm{D}$ configuration, the value of the linear criteria $T_{c}$ would be fixed by the geometric parameter $\Gamma$, as plotted in Fig. 3.



(a)

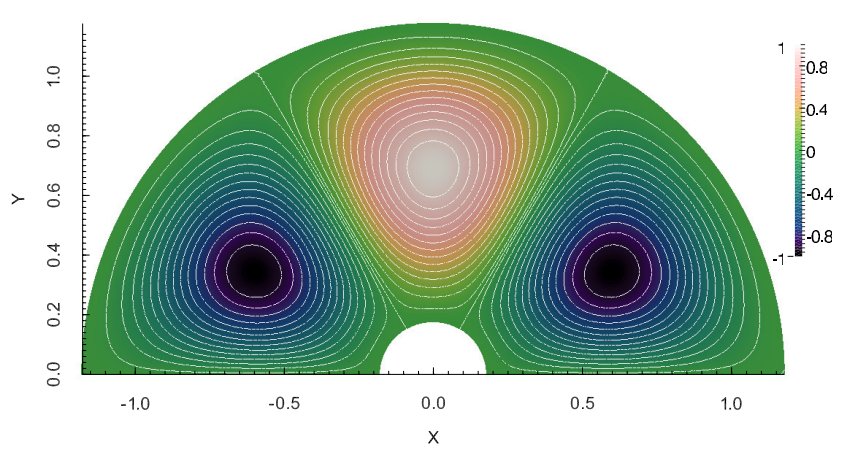

(b)

FIG. 5. Stream function contour plots of the eigenfunctions of the most unstable modes for $\Gamma=0.05$ (a) and $\Gamma=0.15$ (b) for the free-walls case. The number of rolls is related with the number $m$ of the corresponding mode in the modal expansion. 




(a)

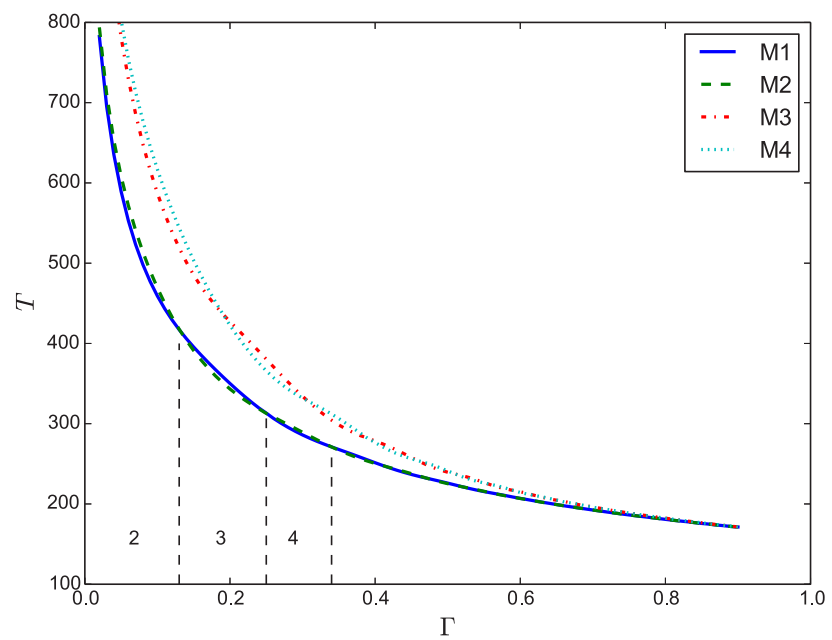

(b)

FIG. 6. First four eigenvalues as a function of $\Gamma$ for rigid walls. Plot (a) corresponds to inner injection, and plot (b) to outer injection. The number of rolls of the dominant mode is showed up to four.

\section{Rigid walls case}

Figure 3 also shows the critical value $T_{c}$ as a function of $\Gamma$ for rigid walls for both inner and outer injection, along with the values for the free wall configurations. In both injection configurations the linear criterion of the free walls case is the lowest one, especially for small values of $\Gamma$. The difference becomes negligible as $\Gamma$ increases above 0.4 . This behavior is due to the viscous shear introduced by the lateral walls in the rigid case. This damping affects only the convective cells in the vicinity of the flat sides. Hence, its influence on the global flow decreases as the number of cells increases, that is, as $\Gamma$ increases. In both curves, humps similar to those present in the free walls configuration are visible, although the cusps are displaced. Each hump corresponds also to a different number of rolls. As $\Gamma$ approaches 1 , the value of $T_{c}$ tends to $T_{c}^{p}=164.1$, the critical value for two infinite parallel plates. The same behavior has already been found for the free walls case. We emphasize that the numbering of the modes does not correspond to the number of rolls appearing in the flow. As $\Gamma$ increases, the first mode develops two lateral smaller rolls. They grow in size and eventually, when the first mode is again the most unstable one, a pattern of three fully developed convective rolls is observed. Then two smaller lateral rolls start to develop and the process repeats itself. A similar behavior is exhibited by the second mode (see Fig. 7).

Figure 6 plots the value of $T$ as a function of $\Gamma$ for the first four most unstable modes for the rigid walls case, for inner and outer injection. Note that the structure of the $T$ versus $\Gamma$ diagram is completely different from that of Fig. 4 . The first and second modes cross each other several times, but they don't cross the other modes. It is the same for the third and fourth modes, and other higher ones. This behavior is similar to what was observed in the 2D rectangular cavity configuration described in Pérez et al. [17], and it can be explained by a similar argument. Figure 7 shows the stream functions of the most unstable mode for $\Gamma=0.05$ and $\Gamma=0.20$ with inner injection. The former corresponds to the first mode and the latter is related to the second one. Both are the most unstable modes for the corresponding values of $\Gamma$. The modes with the same parity have the same symmetry with respect to the $x=0$ axis: antisymmetric for the odd modes and symmetric for the even ones. The paths of the modes with the same symmetry

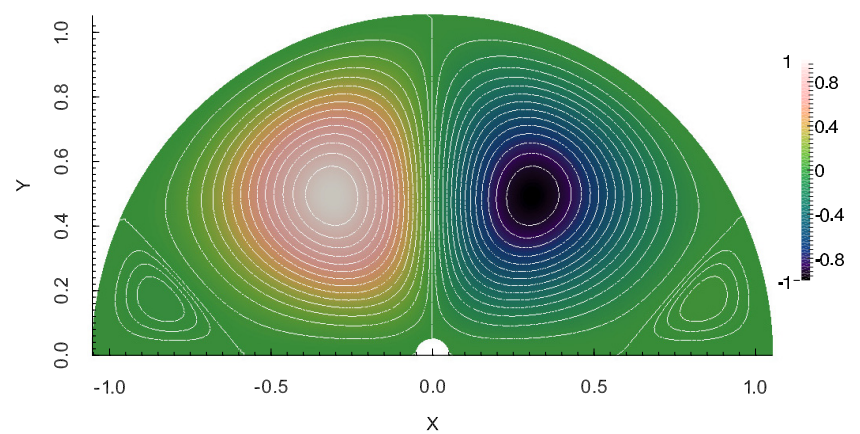

(a)



(b)

FIG. 7. Stream function contour plots of the eigenfunctions of the most unstable modes for $\Gamma=0.05$ (a) and $\Gamma=0.20$ (b) for the rigid walls case and inner injection. The first one is antisymmetric and the second one is symmetric. The counter rolls in the corners appear because of the no-slip boundary conditions for velocity. 




(a)

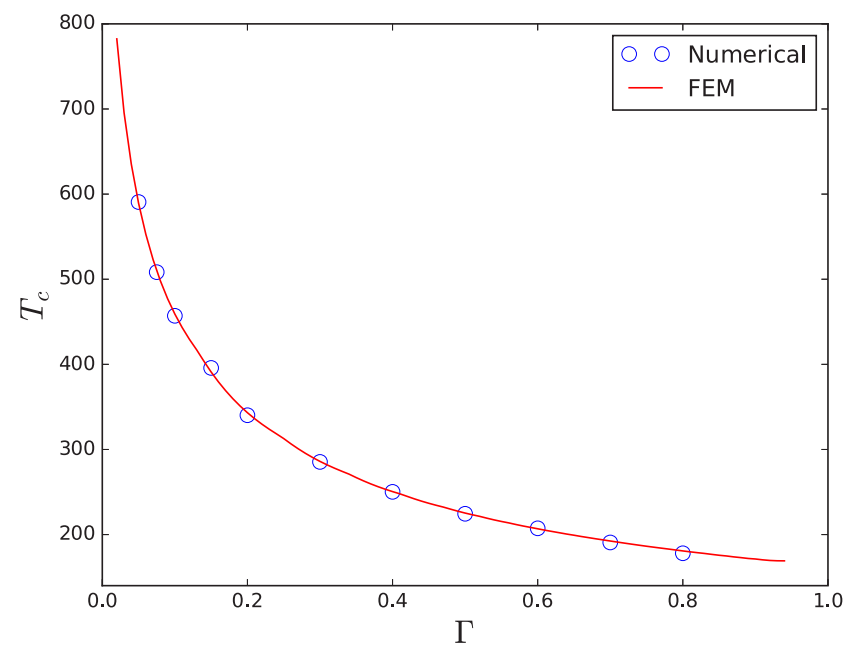

(b)

FIG. 8. Linear stability criteria as a function of $\Gamma$ for $C=10$ for inner (a) and outer (b) injection in the case of rigid walls. The discrete points correspond to values computed from the numerical simulations, while the solid lines have been computed with the FEM-based stability analysis.

do not cross each other, so mode 3 repels mode 1 , and mode 4 repels mode 2 , forcing modes 1 and 2 to cross each other. The mathematical reason is related to the conditions needed to get degenerate eigenvalues in the eigenvalue problem. In general, in a two-parameter system, two paths of symmetrybreaking bifurcation points intersect only if they break different symmetries (or if the solutions belong to different symmetry classes), whereas the path of bifurcation points that break the same symmetry cannot cross one another. For a more complete explanation we refer the reader to our previous paper [17], and also to other works on the Rayleigh-Bénard-Marangoni problem, where this behavior has also been reported (see for example refs. [25-27]).

The free walls case is a degenerate problem, and the modes can cross each other without any restriction. It is worth to not that, while for the free walls configuration all the convective rolls are equivalent, this is not the case for the rigid walls case. The new convective roll in the central region in the plot (b) of Fig. 7 is not a replica of the rolls in plot (a) of the figure. Indeed, this is due to the presence of the counter-rotating rolls close to the corners, induced by the no-slip boundary conditions for velocity.

\section{NONLINEAR ANALYSIS}

In this section, we consider the nonlinear aspects of the problem, in particular the nature of the bifurcation and how it changes with the values of the parameter. The complexity of the mathematical problem makes very difficult to perform an analytic or semianalytic study, similar to what we have done for the linear stability. Therefore, we have solved numerically the full problem defined by the set of Eqs. (4) along with the associated boundary conditions Eqs. (5).

\section{A. Numerical methods}

We solve the set of Eqs. (4) using our in-house code Oracle. This code implements a second order in time and space finite volume method [28]. The computational domain is discretized with a structured grid consisting of nonorthogonal quadrilaterals. All the meshes have 150 cells along the radial direction, but the number of cells along the azimuthal direction increases with the value of $\Gamma$. For example, the mesh is $330 \times 150$ for $\Gamma=0.05$ and $628 \times 150$ for $\Gamma=0.5$. In all the cases, the mesh is thinner near the injecting electrode. We use a colocated arrangement, i.e., all variables are stored at the center of each control volume. The central differencing (CD) scheme and the improved deferred correction (IDC) scheme [29] are used to compute the convective and diffusive fluxes in the NavierStokes equations, respectively. For time integration, we use a second-order semi-implicit three time levels (I3L) scheme [28]. The velocity-pressure coupling algorithm is undertaken by the SIMPLE algorithm [30], as the fluid is assumed to be incompressible.

The charge transport equation is hyperbolic, therefore it requires the use of special methods [31,32]. To prevent the development of spurious numerical oscillations in the charge density distribution, it is recommended to use nonoscillating, nondiffusive, and bounded schemes [33,34]. In this study, we have chosen a TVD scheme, the third-order smooth monotonic algorithm for real transport (SMART) scheme of Gaskell and Lau [35]. We refer to Refs. [31,32] for additional details. The improved least-squares approach [36] is used to compute all gradients including the electric field.

The simulations related to the projection on the modal basis have been made with finite elements using the COMSOL multiphisics software. The numerical computations of the charge transport equation have been made with the transport of diluted species module. This module includes a crosswind stabilization scheme by Do Carmo and Galeao [37]. Although more diffusive than the SMART scheme described above, it controls well the undershoots and overshoots, so that the values of electric charge density remain positive at all times. 


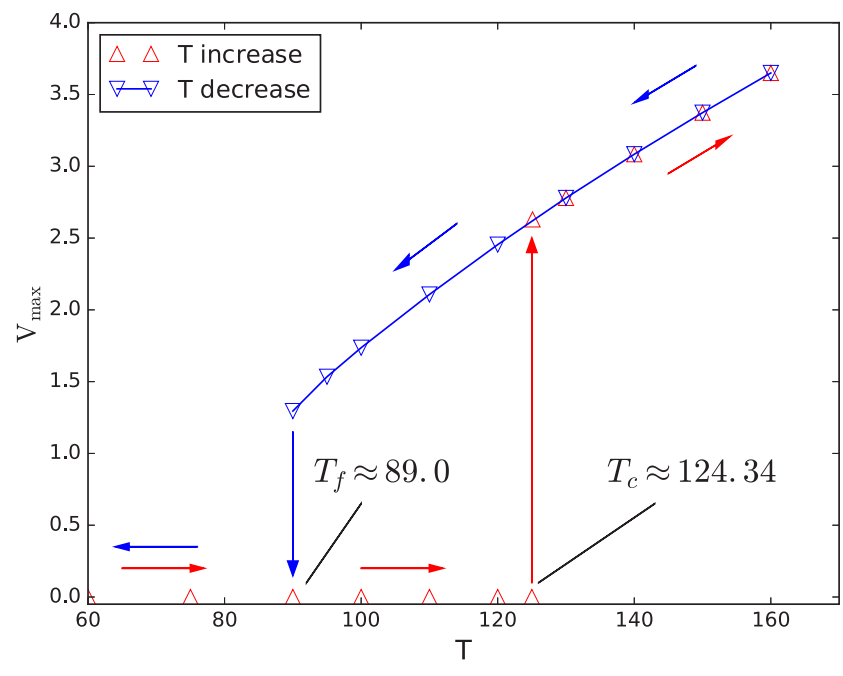

(a)

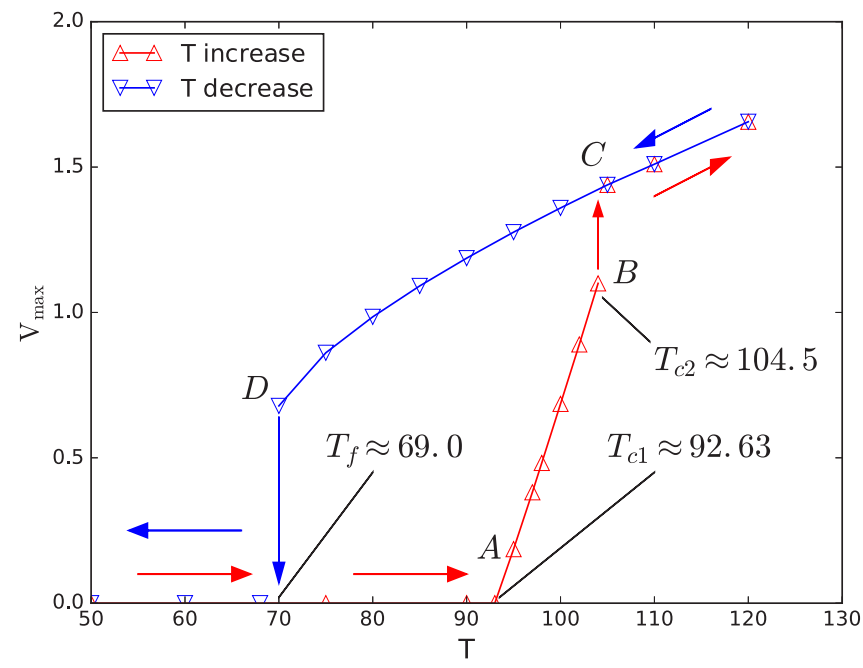

(b)

FIG. 9. Bifurcation diagrams for inner injection, rigid walls, $\Gamma=0.5$ (a) and $\Gamma=0.05(\mathrm{~b})$. The bifurcation is subcritical in the first case and supercritical in the second one.

\section{B. Results}

All the simulations in this paper have been performed with $C=10$ and $M=60$.

\section{Linear stability}

As a validation, we reobtain from the direct numerical simulations the linear stability criteria discussed previously, for inner and outer injection in the case of no-slip walls. We start from the hydrostatic solution of Eqs. (4) and boundary conditions Eqs. (5) for a given value of $\Gamma$. Then we start a simulation computing all the physical magnitudes for different values of $T$ until the onset of fluid motion. Then we obtain the critical value $T_{c}$ for each $\Gamma$ from the growth factor of the perturbations. The procedure is more detailed in $\mathrm{Wu}$ et al. [15].

Figure 8 plots the computed thresholds for several values of $\Gamma$ for inner [Fig. 8(a)] and outer [Fig. 8(b)] injection. The solid lines correspond to the values obtained with the Finite Element (FEM) analysis detailed in Sec. III. The agreement is very good for both cases. The cusps observed in the plot correspond to the crossing of modes described in Sec. III. These cusps are clearer in the inner injection configuration.

\section{Inner injection}

In $\mathrm{Wu}$ et al. [15] we discussed the non linear phenomena associated to two coaxial complete cylinders. The bifurcation in that case was subcritical. This means that, once the value of $T$ is greater than the linear threshold $T_{c}$ and the motion starts, the system jumps to a steady state where the maximum velocity of the fluid is always greater than the maximum drift velocity induced by the electric field. This behavior can also be obtained in the half cylinder configuration. Plot (a) in Fig. 9 shows the bifurcation diagram for $\Gamma=0.5$ and inner injection. For $T>T_{c}=124.34$, the system jumps to the upper branch. If $T$ is further increased, the maximum velocity increases as indicated by the vertical upwards arrow. The figure depicts the typical hysteresis associated to subcritical bifurcations. Once we reach the upper branch, if we start decreasing $T$ the system stays on the upper branch even for values $T<T_{c}$. This goes on down to a nonlinear critical value $T_{f}$ (about 89.0 for this value of $\Gamma$ ). If $T<T_{f}$ the motion stops and the system goes back to the hydrostatic state.

However, the nature of the bifurcation can change for some values of $\Gamma$. Plot (b) in Fig. 9 depicts the bifurcation diagram for inner injection and $\Gamma=0.05$. In that case, we can see that above the linear critical value $T_{c 1}=92.63$ a supercritical stable branch appears. Along this branch we find steady states where the maximum velocity of the fluid is lower than the maximum drift velocity. As the value of $T$ increases we follow this supercritical branch, up to a new critical value $T_{c 2}=$ 104.5, where the system jumps to a finite amplitude branch similar to that one observed for $\Gamma=0.5$. In this branch the maximum velocity of the liquid is greater than the maximum drift velocity. If we decrease now the value of $T$ we follow this branch down to a non linear threshold $T_{f} \simeq 69.0$. Figure 10 shows the electric charge distribution for the stationary states corresponding to points $B(\mathrm{a}), C(\mathrm{~b})$, and $D(\mathrm{c})$ in Fig. 9. The first point is on the supercritical branch, the velocity of the fluid is not high enough to overcome the drift velocity, and there is no region void of charge. Points $C$ and $D$ are on the upper branch. Here, the electric charge distribution in the bulk is mainly controlled by the velocity of the fluid. As a consequence, regions void of charge are formed in the bulk.

Our simulations show that the supercritical branch only exists for small values of $\Gamma$. We can define three critical values of parameter $T: T_{c 1}$ is the linear criterion to start the motion of the liquid; $T_{c 2}$ the critical value at which the system jumps from the supercritical to the upper branch; $T_{f}$ is the non linear criterion at which the motion of the liquid stops when we follow down the upper branch. Figure 11 plots these critical values for several values of $\Gamma$. We have not found any values of $T_{c 2}$ beyond $\Gamma=0.125$. The bifurcation is subcritical for higher values of the radius ratio and supercritical for smaller ones. In all cases, the values of $T_{f}$ are smaller than the corresponding $T_{c 1}$, as it is expected from the bifurcation structure. 
(a)

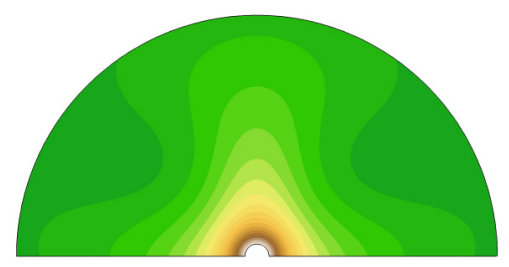

(b)

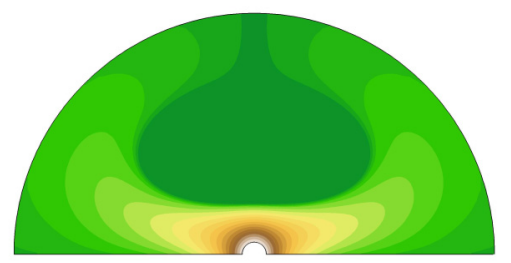

(c)

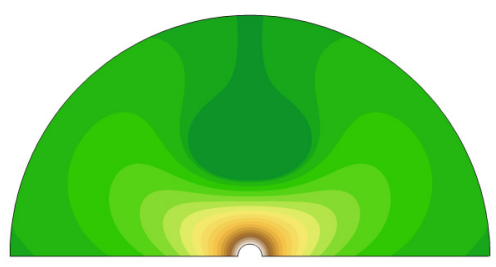

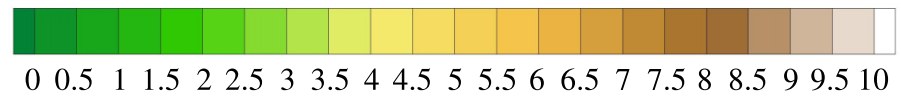

FIG. 10. Electric charge density for rigid walls, inner injection, $\Gamma=0.05$ and points $B(\mathrm{a}), C(\mathrm{~b})$, and $D(\mathrm{c})$ in Fig. 9 . Point $B$ is on the supercritical branch and points $C$ and $D$ are on the subcritical branch.

The switch from subcritical to supercritical is induced by the presence of the no-slip boundary conditions at the flat sides. Figure 12 shows the distributions of electric charge and the velocity field for three different cases. Plots (a1) and (a2) correspond to the free walls case, for $T=75$. This problem is similar to the whole cylinders geometry. The bifurcation is subcritical [15], similar to the one showed in the plot (a) of Fig. 9. For this value of $T$ the system is on the nonlinear branch, with the flow completely developed. We can see the central void region with no electric charge, created by the competition between the electric drift and the velocity of the flow. Plots (b1) and (b2) show the charge distribution and the velocity field for the rigid case for $T=98$. This point is on the supercritical branch (point $A$ in Fig. 9). Here, the velocity of the liquid only perturbs slightly the hydrostatic solution. We can see the two big counter-rotating rolls in the velocity field created by the no-slip boundary condition. These counter-rotating rolls are not present in the free walls configuration. There are also two very small counter-rotating rolls at the inner corners, although

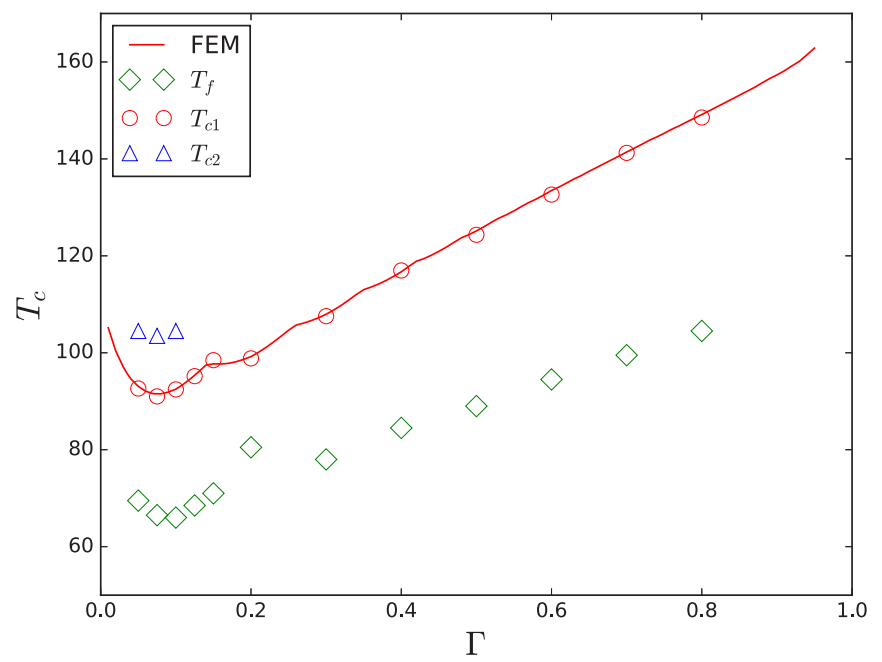

FIG. 11. Critical values of parameter $T . T_{c 1}$ is the linear criteria, $T_{c 2}$ is the value where the system jumps to the nonlinear branch, $T_{f}$ is the minimum value to have motion of the fluid. The solid line shows the linear criteria computed with FEM. $T_{c 2}$ only exists for small values of $\Gamma$. they are difficult to see. Plots (c1) and (c2) correspond to point $C$ in Fig. 9. Here we are on the finite amplitude branch and the velocity of the fluids creates the void region in the center. The counter-rotating rolls can also be seen, but they are smaller than in plot (b2).

\section{Outer injection}

Figure 13 shows the bifurcation diagram for outer injection, rigid walls and $\Gamma=0.05$. The bifurcation is subcritical for all the values of $\Gamma$ that we have explored. The linear critical threshold obtained from the computations is $T_{c}=589.4$, to be compared with the value obtained from the eigenvalue analysis $\left(T_{c}^{\mathrm{eig}}=589.3\right)$. This highlights the excellent agreement between the two methods employed to determine the linear stability criteria. For $T>T_{c}$ the system jumps directly to the nonlinear branch. This branch is shorter than the equivalent one in the inner injection case. For $T \geqslant T_{f 2}=630$ a new instability appears. The two-roll pattern becomes unstable and an oscillation between a two-roll pattern and a four-roll pattern occurs. Figure 14 shows the charge density and the stream functions for $T=345$ (a) and $T=600(\mathrm{~b})$. The second value corresponds to a state slightly above the critical value $T_{c}$ and the first one to one state slightly above the critical value $T_{f} \simeq 344$. These two plots are equivalent to the plots (b) and (c) in Fig. 10. As we follow down the nonlinear branch the void region shrinks, until the electric torque cannot sustain the motion anymore.

\section{Modal analysis}

The main difference between the free and rigid walls case is the presence of the counter-rotating rolls in the corners, induced by the no-slip boundary conditions. This affects the flow pattern and, consequently, the charge distribution. We can try to understand the physical differences between these two configurations by performing a modal analysis of the flow. In Sec. III we computed numerically the eigenfunctions corresponding to the rigid walls case. These eigenfunctions can be used as a base to approximate the velocity field:

$$
\boldsymbol{u}^{r}(x, y, t)=\sum_{j=1}^{N} A_{j}^{r}(t) \boldsymbol{u}_{j}^{r}(x, y)
$$




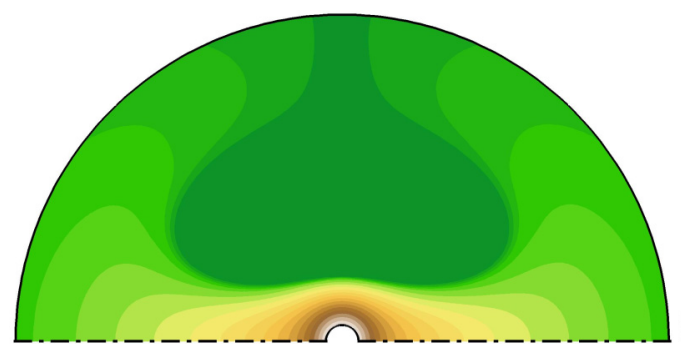

(a1)



(b1)

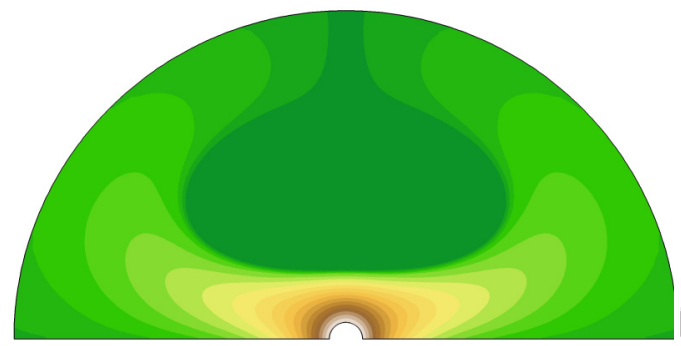

(c1)



(a2)



(b2)



(c2)

FIG. 12. Electric charge density (1) and velocity field (2) for $C=10, \Gamma=0.05, M=60$ : (a) free case with $T=75$, (b) rigid walls with $T=98$, (c) rigid walls with $T=105$.

Here $\boldsymbol{u}^{r}(x, y, t)$ is the velocity field of the fluid in the rigid walls case, $\boldsymbol{u}_{j}^{r}(x, y)$ are the eigenfunctions obtained with the linear stability analysis, and $A_{j}^{r}(t)$ are the amplitudes of the modes. The number $N$ is the number of modes retained in the approximation.

We introduce this expansion in the Navier-Stokes equations [first equation in Eqs. (4)]. Then we multiply by the eigenfunction $\boldsymbol{u}_{i}^{r}(x, y)$ and integrate over the domain. In this way we obtain ordinary differential equations which govern the time evolution of the modes amplitudes:

$$
B_{i j}^{r} \frac{d A_{j}^{r}}{d t}=\frac{M^{2}}{T} K_{i j}^{r} A_{j}^{r}+M^{2} I_{j}^{r} .
$$

The matrices and vectors in this expression are

$$
\begin{aligned}
B_{i j}^{r} & =\int_{\Omega} \boldsymbol{u}_{i}^{r}(x, y) \cdot \boldsymbol{u}_{j}^{r}(x, y), \\
K_{i j}^{r} & =\int_{\Omega} \boldsymbol{u}_{i}^{r}(x, y) \cdot \Delta \boldsymbol{u}_{j}^{r}(x, y) \\
& =-\int_{\Omega} \nabla \boldsymbol{u}_{i}^{r}(x, y): \nabla \boldsymbol{u}_{j}^{r}(x, y), \\
I_{j}^{r}(t) & =\int_{\Omega} q^{r}(x, y, t) \boldsymbol{E}^{r}(x, y, t) \cdot \boldsymbol{u}_{j}^{r}(x, y) .
\end{aligned}
$$

The integrals involving the pressure gradient vanish when integrating by parts and applying the boundary conditions fulfilled by the eigenfunctions. We apply a similar procedure on the elements of $K_{i j}^{r}$. The elements of $B_{i j}^{r}$ and $K_{i j}^{r}$ are constants,

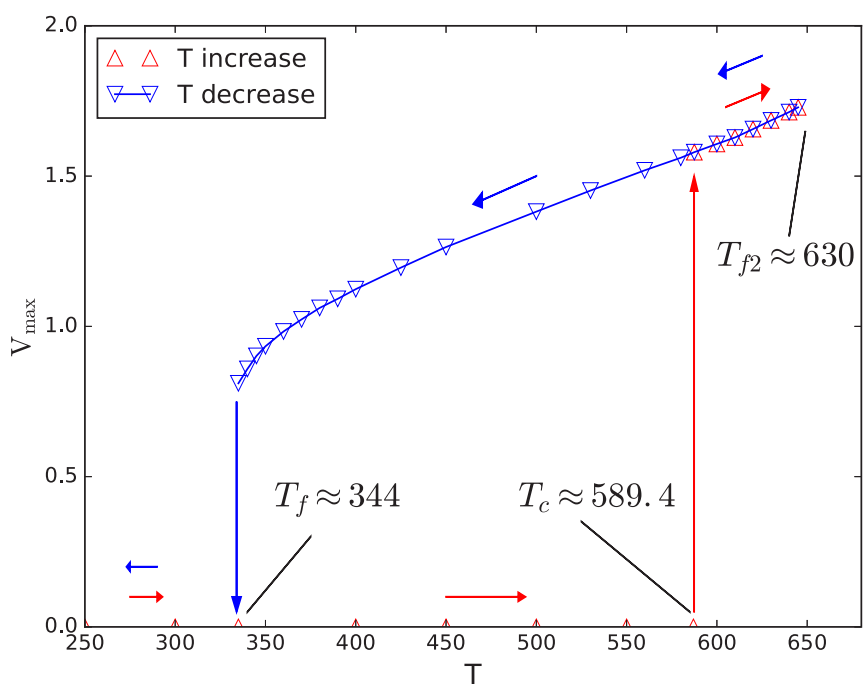

FIG. 13. Bifurcation diagram for outer injection, rigid walls, $\Gamma=$ 0.05 . The bifurcation is subcritical for all the explored values of $\Gamma$. 




(a)

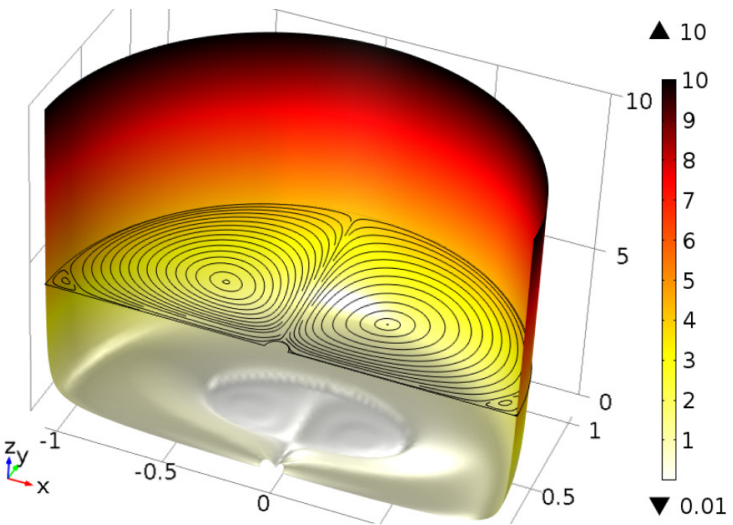

(b)

FIG. 14. Charge density distribution and streamlines for outer injection, $T=345$ (a) and $T=600$ (b). The axis with the $z$ label in the plots corresponds to the value of the charge density.

but the elements of $I_{j}^{r}$ change with time. Multiplying Eq. (18) by the inverse of $B_{i j}^{r}$ we obtain a set of equations for the temporal derivative of the amplitudes of the modes:

$$
\frac{d A_{j}^{r}}{d t}=\frac{M^{2}}{T} C_{i j}^{r} A_{j}^{r}+M^{2}\left(B_{i j}^{r}\right)^{-1} I_{j}^{r} .
$$

The first term in the right-hand side of Eq. (20) represents the effect of viscosity. It will always have a negative contribution to the evolution of the amplitude. The second term is related to the power given to the modes by the electric field. A similar expansion can be used for the free walls case using the eigenfunctions for free-slip boundary conditions at the flat sides. The velocity can be approached by

$$
\boldsymbol{u}^{f}(x, y, t)=\sum_{j=1}^{N} A_{j}^{f}(t) \boldsymbol{u}_{j}^{f}(x, y) .
$$

Here, the $f$ stands for the free walls case. Applying the same steps we get

$$
\frac{d A_{j}^{f}}{d t}=\frac{M^{2}}{T} C_{i j}^{f} A_{j}^{f}+M^{2}\left(B_{i j}^{f}\right)^{-1} I_{j}^{f} .
$$

The definitions of $B_{i j}^{f}, K_{i j}^{f}$ and $I_{j}^{F}$ are similar to those given in Eq. (19), taking the fields from free walls instead of those from rigid ones.

There is an important difference between the free and rigid walls configuration. The elements of $B_{i j}^{f}$ and $B_{i j}^{r}$ are the dot products of the modal functions for each configuration. Table I shows the values of the dot products between the first mode and the others for $\Gamma=0.05$ and for both free and rigid walls. The modal functions are normalized so that, for each one of them, $\int_{\Omega}\left|\boldsymbol{u}_{i}^{f, r}\right|^{2}=1$. In the free case the other modes are essentially orthogonal to the first one, that is, the dot products are almost zero. For rigid walls this is not the case, as three other modes have a non negligible dot product with the first one, especially the modes six and seven. As $\Gamma$ increases the modes for the no-slip configuration becomes more orthogonal, as it is shown in the table. This nonorthogonality will have consequences in the time evolution of the system, as we will see later.
The computational results related to the modal analysis presented in this section have been performed with COMSOL Multiphysics. The mesh had 9600 triangles and 2240 quadrangles. The interpolation functions were of order 1 for velocity, pressure and charge density, and order 2 for the electric potential. The mesh was especially refined near the boundaries of the domain, where several boundary layers of quadrangles were added. This is done to better account for high gradients in this zone.

Figure 15 shows the time evolution of the amplitudes of the first eight modes for three cases in the inner injection configuration. The upper plot corresponds to the free walls case and $T=70$. Here, the bifurcation is subcritical. We can observe the fast growth of the first mode within the time interval $(3,10)$, approximately. Due to the orthogonality of the eight first modes, in this region the first mode grows undisturbed by the others ones, until the velocity field takes control of the charge distribution, originating the typical void region in the subcritical branch. Plot (b) corresponds to rigid walls with $T=95$. Here we lay on the supercritical branch. The modes are not orthogonal, so several modes get excited, competing for the energy transferred by the electric field. None of the modes gets clearly ahead of the others, and the final velocity field is not strong enough to create the void region. The lower plot corresponds to inner rigid walls with $T=105$. Here, we lay on the upper branch. The behavior of the amplitudes at early times is similar to the one observed for $T=95$. The main difference is the evolution of the amplitude of the second mode. This value of $T$ is higher than the excitement threshold for this second mode $(T=104)$. As this mode is excited, it becomes dominant, although eventually it fades among the others modes, especially the modes 1 and 7 , which are dominant in the supercritical branch. So, even if the second mode is not the dominant one in the final state, its appearance changes radically the behavior of the dominant modes in the supercritical branch, pushing the system to fall on the upper branch. This is consistent with the value $T_{c 2}=104.5$ in Fig. 9 corresponding to the jump from the supercritical to the upper branch. This jump occurs for a value of $T$ close to the excitation threshold of the second mode. 
TABLE I. Values of the dot products $(1, j)=\int_{\Omega} \boldsymbol{u}_{1} \cdot \boldsymbol{u}_{j}$ of the dominant mode with the other modes for rigid and free walls cases. As $\Gamma$ increases, the rigid modes becomes more orthogonal.

\begin{tabular}{cccccccccc}
\hline \hline & $(1,1)$ & $(1,2)$ & $(1,3)$ & $(1,4)$ & $(1,5)$ & $(1,6)$ & $(1,7)$ & $(1,8)$ \\
\hline \multirow{2}{*}{$\Gamma=0.05$} & Rigid & 1 & $+4 \times 10^{-13}$ & $+6 \times 10^{-13}$ & $-9 \times 10^{-02}$ & $-8 \times 10^{-13}$ & $+3 \times 10^{-01}$ & $+8 \times 10^{-01}$ & $+3 \times 10^{-11}$ \\
& Free & 1 & $-2 \times 10^{-10}$ & $+6 \times 10^{-07}$ & $+3 \times 10^{-10}$ & $+4 \times 10^{-10}$ & $+2 \times 10^{-06}$ & $-2 \times 10^{-09}$ & $+2 \times 10^{-09}$ \\
$\Gamma=0.15$ & Rigid & 1 & $+6 \times 10^{-12}$ & $+8 \times 10^{-15}$ & $+2 \times 10^{-02}$ & $-1 \times 10^{-13}$ & $-1 \times 10^{-02}$ & $+2 \times 10^{-01}$ & $-3 \times 10^{-12}$ \\
& Free & 1 & $+2 \times 10^{-11}$ & $+2 \times 10^{-10}$ & $-2 \times 10^{-07}$ & $+1 \times 10^{-10}$ & $+4 \times 10^{-07}$ & $-2 \times 10^{-09}$ & $+4 \times 10^{-10}$ \\
\hline \hline
\end{tabular}

The influence of the second mode is also consistent with the similar behavior observed in the rectangular configuration with rigid walls described in $\mathrm{Wu}$ et al. [16]. In that configuration,
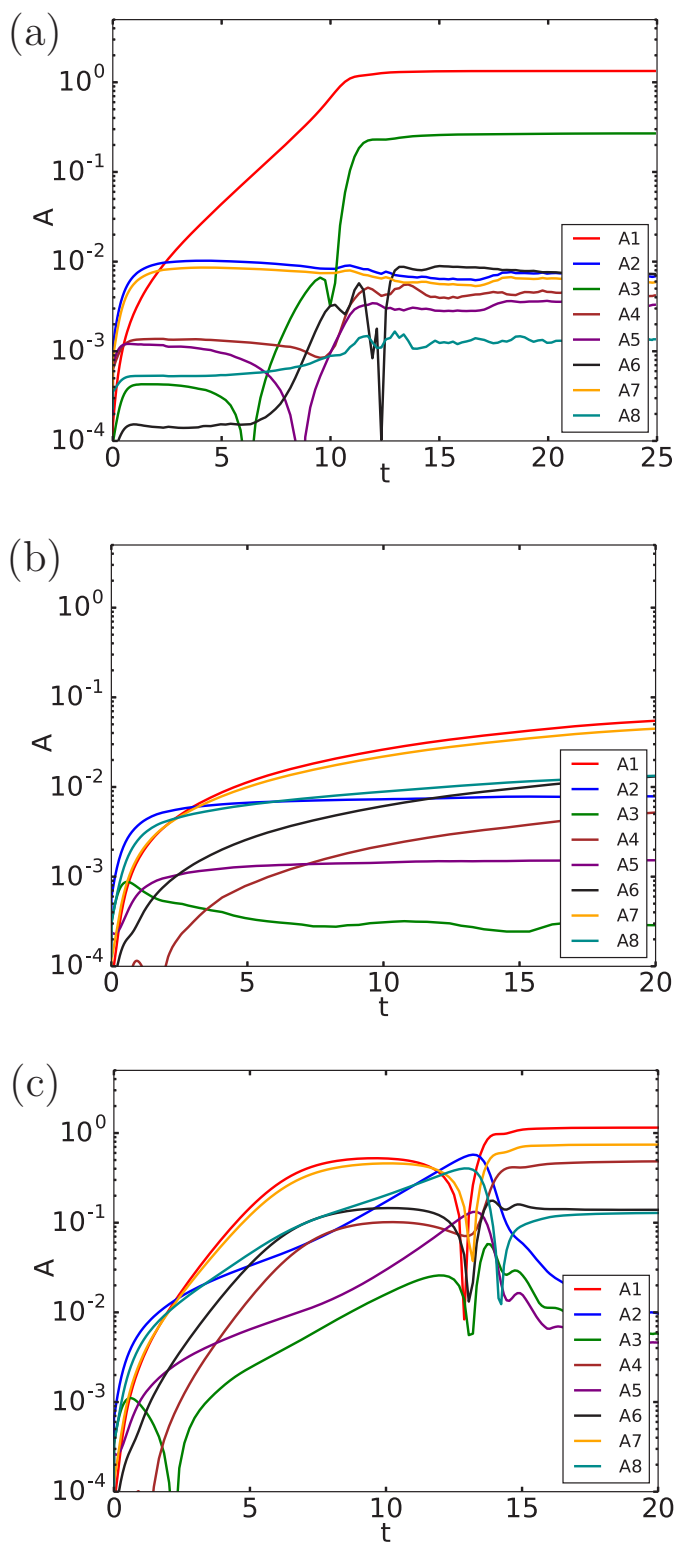

FIG. 15. Amplitudes of the time evolution of the first eight modes for inner injection with $\Gamma=0.05, C=10$, and $M=60$. Plot (a) corresponds to free walls and $T=70$, plot (b) to rigid walls and $T=95$, and plot (c) to rigid walls and $T=105$. The vertical axis are plotted in logarithmic scale. the modes also cross each other as the aspect ratio of the rectangular domain increases. For values of the aspect ratio below the first crossing, the bifurcation is supercritical. As the aspect ratio is increased, it becomes subcritical for a range of values of the aspect ratio around the one corresponding to the first crossing of modes. Then it becomes supercritical again until the next crossing, where it turns subcritical and stays so for further values of the aspect ratio. Near the crossings, the second mode is excited, as it happens in the half-cylinder case, and it throws the system away from the supercritical branch into the finite amplitude one. Beyond the second crossing, the influence of the lateral rigid walls on the flow patterns of the modes becomes negligible, and the supercritical branch disappears completely. In the half cylinders configuration we do not observe the reappearance of the supercritical branch after the first crossing. But there is a difference with the rectangular case. Here, as the ratio between the radii increases, the value of $T_{c}$ increases, while in the rectangular configuration $T_{c}$ decreases as the aspect ratio increases. With higher value of $T_{c}$, more energy is injected into the modes, and they grow until the velocity field takes control of the electric charge distribution, producing the region void of charge, a typical characteristic of the subcritical bifurcation.

For the outer injection no supercritical branch has been observed in the numerical simulations that we have carried out. The same considerations about the orthogonality of the modes apply in the outer injection configuration. But there is a mayor difference, the value of $T_{c}$ is much higher here. Hence, the input of electric energy is also much higher. Even if the dominant mode faces the competition with other modes in the rigid walls case, it can grow because the quantity of energy available is much greater than in the inner injection configuration. Figure 16 plots the time evolution of the amplitude of the first eight modes for outer injection with $\Gamma=0.5, C=10$, and $M=60$. Plot (a) corresponds to the free walls case with $T=500$, just above the linear threshold. Plot (b) corresponds to the rigid walls configuration with $T=600$, also just above the critical threshold. The plot for the free-walls configuration is quite similar to the same case with inner injection (upper plot in Fig. 15). The second mode is dominant at earlier times, but once the first mode starts growing, it does not stop until it becomes the dominant one. For the rigid configuration, the first mode is the dominant one in the growing region, but other three modes get amplitudes not too far from the dominant one. The difference with the inner injection case [plot (b) in Fig. 15] is that the growth does not stop until the amplitudes are high enough to sustain a void region in the bulk, producing the subcritical bifurcation. This is due to the high value of the parameter $T$, compared with the value on the supercritical 


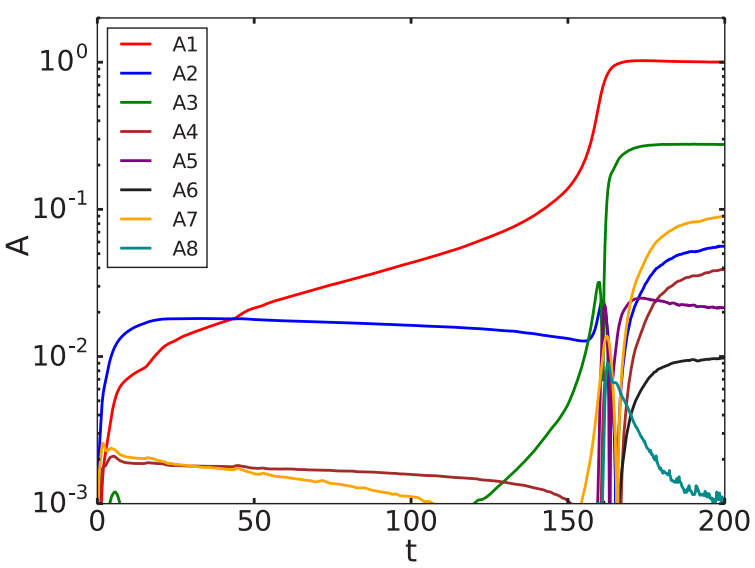

(a)



(b)

FIG. 16. Amplitudes of the time evolution of the eight first modes for outer injection with $\Gamma=0.05, C=10$ and $M=60$. Plot (a) corresponds to free walls and $T=500$ and plot (b) to rigid walls and $T=600$. The vertical axis are plotted in logarithmic scale.

branch in the inner injection case, which is much lower. In the outer injection we do not observe the disruption produced by the second mode on the supercritical branch, as this one does not exist. Let us also stress that in this case there is no dominant mode. When the flow is fully developed, there are at least three relevant modes of similar intensity. This explains why the upper branch is shorter in the outer injection configuration. The excited modes compete with each other and eventually destabilize the branch.

\section{CONCLUSIONS}

In this paper we have discussed the linear stability and the nature of the nonlinear bifurcation of the injection induced electroconvection in a perfectly insulating liquid between two half cylinders with no-slip conditions on all boundaries. This is a variation of the classical EHD problems of electroconvection between two concentric cylinders.

Due to the no-slip boundary conditions the usual modal analysis of the linear stability is not applicable. Therefore, we have chosen an alternative strategy, based on the finite element method, already used in a similar problem with a rectangular rigid container. First, we have considered as a validating test case a problem with the same geometry and with symmetry conditions on the flat sides. The linear stability analysis of this problem is equivalent to the full cylinders case, and can be solved with the standard modal analysis. The agreement between the results obtained with the standard modal approach and the finite element technique used here is excellent.

We have shown that the linear critical value is higher when the no-slip conditions are imposed, as a consequence of the viscous shear induced by the rigid walls, although this effect decreases as the ratio $\Gamma=R_{i} / R_{o}$ between the cylinder radii approaches 1 . We also find that the structure of the paths traced by the modes in the $\Gamma-T$ diagram in the rigid case differs from the structure for the free case. In the latter all the modes crosses each other, while in the former a repulsion appears between the modes with the same symmetry, forcing the first and second modes to cross each other several times. This behavior occurs in both inner and outer injection. The difference is that for outer injection the value of the linear criterion is much higher than in the inner injection case.

When the free-slip boundary conditions are imposed on the flat side walls the nonlinear bifurcation is always subcritical. In the final state the distribution of electric charge is controlled by the velocity field, originating a region in the bulk void of electric charge. When the no-slip boundary conditions are imposed on the flat walls, the bifurcation becomes supercritical in some configurations. In this case, in the final state the velocity field is not strong enough to overcome the electric field and no void region develops in the bulk.

The bifurcation becomes supercritical when the injection occurs on the inner cylinder and the ratio between the outer and inner cylinder radii is small. Here, there are three critical values of the stability parameter. If the electric field is increased beyond the linear threshold, $T_{c 1}$, the velocity field is not strong enough to overcome the electric field. Hence, the distribution of electric charge is the same than in the hydrostatic regime perturbed by the velocity, and there is no void region. In this case the bifurcation is supercritical. As the electric field is further increased, when $T=T_{c 2}>T_{c 1}$ the system jumps to the finite amplitude branch, where the velocity controls the charge distribution and a region void of charge appears. If the electric field is now decreased the velocity is sustained until a nonlinear threshold $T_{f}<T_{c 1}$ is reached. For $T<T_{f}$ the velocity disappears and the charge distribution is the hydrostatic one. For ratios $\Gamma \gtrsim 0.125$ the supercritical branch disappears and the system jumps directly to the upper branch once the flow starts. When the injection occurs at the outer electrode the bifurcation is always subcritical.

To understand the change in the nature of the bifurcation, we have performed a modal analysis using the eigenfunctions computed from the linear analysis as a modal basis. The modes are orthogonal in the free-slip configuration, while this is not the case in the no-slip configuration. For the inner injection case and the free-slip configuration, with a subcritical bifurcation, the dominant mode grows undisturbed until it controls the charge distribution. In the rigid case, on the supercritical branch $\left(T_{c 1}<T<T_{c 2}\right)$, the nonorthogonality of the modes implies 
that several modes are able to extract energy from the electric field at the same time. As a consequence, several modes of similar intensity are relevant in the flow, disturbing each other and none of them dominates. The final velocity field is not strong enough and no void region is formed, preventing the occurring of a subcritical bifurcation. If $T$ is further increased, the second mode eventually becomes excited. The presence of this second mode disturbs the growth of the other modes. It becomes dominant at a certain time and, while it is overcome by the other modes at the final state, its presence changes the distribution of electric charge and pushes the system to the subcritical branch. As the ratio of radii increases, the modes become more orthogonal, since more velocity rolls develop and the influence of the counter-rotating rolls diminishes. As a consequence, the bifurcation is subcritical, same as in the free-slip case.

For the outer injection regime, the modal analysis shows that the amplitudes of the modes grow very fast. Thus, even if the modes are not orthogonal, the velocity field overcomes the driving of the electric field and develops a region void of charge, originating a subcritical bifurcation. The reason that could explain the difference of behavior between the outer and inner injection configurations has to be ascribed to the value of the linear threshold $T_{C}$, which is much higher in the outer injection configuration. Hence, more energy is made available for all the modes to grow and no supercritical branch exists.

These mechanisms explain also the previously unexplained appearance of the supercritical branch in the closed rectangular configuration described in reference Pérez et al. [17].

\section{ACKNOWLEDGMENTS}

This work has been carried out with financial support from the Spanish Ministerio de Economía y Competitividad under Research Project No. FIS2014-54539-P (to P.A.V. and A.T.P.). J.W. acknowledges the Fundamental Research Funds for the Central Universities (Grant No. AUGA5710051817). P.T. acknowledges the support by the French government program Investissements d'avenir (Labex interactifs, Référence No. ANR-11-LABX-0017-01) and a grant from the French district Poitou-Charentes.
[1] J. Seyed-Yagoobi, J. Electrostat. 63, 861 (2005).

[2] B. D. Iverson and S. V. Garimella, Microfluid. Nanofluid. 5, 145 (2008).

[3] J. C. Ryu, H. J. Park, J. K. Park, and K. H. Kang, Phys. Rev. Lett. 104, 104502 (2010).

[4] F. M. J. McCluskey, P. Atten, and A. T. Pérez, Int. J. Heat Mass Transf. 34, 2237 (1991).

[5] J. Seyed-Yagoobi and J. E. Bryan, Adv. Heat Transfer 33, 95 (1999).

[6] S. Laohalertdecha, P. Naphon, and S. Wongwises, Renewable Sustainable Energy Rev. 11, 858 (2007).

[7] M. Jalaal, B. Khorshidi, and E. Esmaeilzadeh, Chem. Eng. J. 219, 118 (2013).

[8] A. Jaworek and A. Krupa, J. Aerosol Sci. 30, 873 (1999).

[9] D. P. H. Smith, IEEE Trans. Ind. Appl. IA-22, 527 (1986).

[10] J. Darabi, M. Ohadi, and D. L. DeVoe, J. Microelectromech. Syst. 10, 98 (2001).

[11] A. Castellanos, A. Ramos, A. González, N. G. Green, and H. Morgan, J. Phys. D 36, 2584 (2003).

[12] A. Jaworek and A. T. Sobczyk, J. Electrost. 66, 197 (2008).

[13] N. Agräit and A. Castellanos, Phys. Fluids A 2, 37 (1990).

[14] D. V. Fernandes, H.-D. Lee, S. Alapati, and Y. K. Suh, J. Mech. Sci. Technol. 26, 3785 (2012).

[15] J. Wu, P. A. Vázquez, P. Traoré, and A. T. Pérez, Phys. Fluids 26, 124105 (2014).

[16] J. Wu, P. Traoré, P. A. Vázquez, and A. T. Pérez, Phys. Rev. E 88, 053018 (2013).

[17] A. T. Pérez, P. A. Vázquez, J. Wu, and P. Traoré, J. Fluid Mech. 758, 586 (2014).

[18] A. Castellanos, IEEE Trans. Electr. Insul. 26, 1201 (1991).

[19] N. Felici, Revue General d'Electrostatique 78, 717 (1969).

[20] P. Atten and R. Moreau, Journal de Mécanique 11, 471 (1972).
[21] A. Logg and G. N. Wells, ACM Trans. Math. Software 37, 20 (2010).

[22] A. Logg, K. A. Mardal, and G. N. Wells, Automated Solution of Differential Equations by the Finite Element Method (Springer, Berlin, 2010).

[23] V. Hernandez, J. E. Roman, and V. Vidal, ACM Trans. Math. Software 31, 351 (2005).

[24] J. Kierzenka and L. Shampine, ACM Trans. Math. Software 27, 299 (2001).

[25] K. A. Cliffe, T. J. Garratt, and A. Spence, SIAM J. Matrix Anal. Appl. 15, 1310 (1994).

[26] K. H. Winters, T. H. Lesser, and K. A. Cliffe, Physica D: Nonlin. Phenom. 29, 387 (1988).

[27] A. van de Vooren and H. Dukstra, Comput. Fluids 17, 467 (1989).

[28] J. H. Ferziger and P. M, Computational Methods For Fluid Dynamics (Springer, Berlin, 2002).

[29] P. Traoré, Y. M. Ahipo, and C. Louste, J. Comput. Phys. 228, 5148 (2009).

[30] S. Patankar and D. Spalding, Int. J. Heat Mass Transf. 15, 1787 (1972).

[31] P. Traoré and A. T. Pérez, Phys. Fluids 24, 037102 (2012).

[32] J. Wu, P. Traoré, and C. Louste, J. Electrostat. 71, 319 (2013).

[33] R. J. LeVeque, Finite Volume Methods for Hyperbolic Problems (Cambridge University Press, Cambridge, 2002).

[34] R. J. LeVeque, SIAM J. Numer. Anal. 33, 627 (1996).

[35] P. H. Gaskell and A. K. C. Lau, Int. J. Numer. Methods Fluids 8, 617 (1988).

[36] D. Mavriplis, AIAA Paper, pp. 2003-3986 (2003).

[37] E. G. D. D. Carmo and A. C. Galeão, Comput. Methods Appl. Mech. Eng. 88, 1 (1991). 